\title{
A propos du «programme systématique» d'abandon des tâches
}

Dernièrement, le directeur de l'Office fédéral de la santé publique (OFSP) était l'hôte du Comité central. L'échange de vues fut très vivant et l'entretien a bien sûr porté, entre autres, sur les questions de prévention et le projet de mise sur pied d'une loi en bonne et due forme sur la prévention. Il semble que l'on s'active dans ce sens à l'OFSP.

Ces travaux administratifs bénéficient d'assez de temps, d'argent et de personnel. En revanche, l'office doit procéder dans d'autres domaines à des économies de postes (45!). La visite s'est terminée avant que le problème de la fermeture de la section «Santé et environnement» ait été abordé. Mais la raison d'une telle mesure n'est pas difficile à deviner, tant est grande la volonté d'économies du chef du DFI.

Quand je pense néanmoins à ce qu'il a fallu pour que des politiques, en relation avec l'augmentation des poussières fines, aient le courage de se prononcer pour une réduction de la vitesse sur les routes ... Quand je pense aussi aux déclarations du nouveau directeur de l'Office fédéral de l'environnement qui, lors d'une émission de radio, ne voulait pas entendre parler de réduction de la vitesse dans ce contexte, se contentant de mesures techniques liées à l'obligation d'équiper les nouveaux véhicules diesel de filtres à particules, l'angoisse m'étreint. Que fait-on d'une réflexion par delà l'entreprise pour laquelle on travaille? Pourquoi est-il si difficile, dans ce genre d'intérêts, d'avoir une pensée plus globale au lieu de ne considérer que les aspects financiers? Doit-on accepter que dans le domaine de la protection ou de la promotion de la santé, on ne connaisse pour objectif que la formule «business for business is business», celle, semble-t-il, des économistes patentés?

Les Médecins en faveur de l'environnement (MfE) lancent une pétition contre la fermeture de la section «Santé et environnement» à l'OFSP. Référez-vous à l'article sur le sujet dans le présent numéro. «La patience vient à bout de tout» dit l'adage: une participation massive à cette pétition devrait ouvrir les yeux du monde politique et l'aider à penser plus loin que le bout de son nez avant d'accepter des mesures qui apportent, certes, des économies à court terme mais qui, à long terme, peuvent avoir des conséquences plus qu'indésirables.

Les formules de pétition à signer peuvent être obtenues auprès de MfE à l'adresse de courriel suivante: info@ aefu.ch.

Ursula Steiner-König, membre du Comité central de la FMH, Domaine prévention et santé 